\title{
Interface instability analysis induced from the flow field in the initial nonuniform characteristics
}

\author{
J. Bai \& T. Wang \\ National Key Laboratory of Shock Wave and Detonation Physics (LSD), \\ Institute of Fluid Physics, China Academy of Engineering Physics, \\ Mianyang, Sichuan, $P$ R China
}

\begin{abstract}
We numerically simulate the previous instability experiments of a double mode perturbed interface in the initial nonuniform flows in an unreshocked case, the numerical results are in very good agreement with the experiment, meanwhile investigate the effect of the nonuniformity of flows on the evolution of instability in nonlinear regime after reshock, by adopting two different nonuniform coefficients $\left(\delta_{1}=0.6162\right.$, and $\left.\delta_{2}=0.4961\right)$ in the Gaussian distribution of the initial nonuniform density. The results indicate that the nonuniformity of the initial flow has great effect on the evolution of instability in the linear and weak nonlinear regime prior to reshock.

Keywords: nonuniform flows, Richtmyer-Meshkov instability, mixing, reshock.
\end{abstract}

\section{Introduction}

The Richtmyer-Meshkov instability occurring at the corrugated interface between two fluids of different densities is of contemporary interest in many fields of research, among which are the inertial confinement fusion (ICF) [1], the fuel mixing in a Scramjet [2] and the explosion of supernova [3]. When an incident shock proceeds into a perturbed interface, vorticity is deposited by the baroclinic torque vorticity production term $\nabla \rho \times \nabla p / \rho^{2}$.

Following the first interaction between the corrugated interface and the shock, a transmitted shock proceeds into the second heavy fluid, and reflects from the end wall of the shock tube, then encounters the evolving layer again. After the 
process called reshock, a reflected rarefaction wave returns into the second fluid and will again interacts with the evolving layer when reflecting from the endwall, producing a compression wave. The compressibility and nonlinearity effects in wave-interface interactions significantly affect the growth rate, making the analytical studies of the growth rate harder.

Numerous investigations focusing on the growth rate after reshocks have been presented. Richtmyer [4] originally confirmed that the growth rate of the initial small perturbation amplitude in single-mode RMI is linearized with time in 1960. Mikaelian [5] explored the growth rate of the interface by applying the potential flow model to growing perturbation to combine the initial and asymptotic stages. Zhang and Sohn [6] presented a derivation of nonlinear theory of Richtmyer-Meshkov instability using Padé approximation from early to later times. Recently, a number of studies investigate the effects of initial conditions on growth rate. Thornber et al. [7] showed that the effects of initial conditions will diminish after a few reshocks because of the change of the form of the perturbation power spectrum. Schilling et al. [8] used the ninth-order WENO shock-capturing method to investigate the late-time mechanism of reshock and mixing, firstly describing the reshock process, and confirmed that the reflected rarefaction has an important role in breaking symmetry and approaching latetime statistical isotropy of the velocity field. Besides, Hill et al. [9] indicated that the reflected rarefaction drives the growth rate of the mixing layer more significantly than the reshock by examining the turbulent kinetic energy. Ukai et al. [10] investigated the effect of the initial conditions on the late time growth by studying four different initial configurations of interface numerically, and found that growth rates after reshock have a little dependence on the initial interface geometry. Leinov et al. [11] showed that the growth rate after reshocks is independent of the initial amplitude by placing the rigid end wall at different distances from the initial contact interface in RM instability experiments.

In those literatures published, the initial flows were confined to a uniform flow field, and nobody investigated the interface instability in the initial nonuniform flows. We firstly investigated the effects of the initial nonuniform flows on the evolution of the unreshocked instability with the experiment and numerical simulation of double perturbed interface [12]. And the initial Gaussian density distribution of the nonuniform flows was determined quantitatively by the combination of numerical simulation and experimental phenomena and data. This work is important for the initial conditions setting and experimental data analysis in experimental study of RM instability. Then we investigate the effects of the nonuniformity of the initial flows on the evolution of the mixing layer in RM instability under reshocks. Numerical simulations of RM instability with reshock for two initial nonuniform flows are performed. The quantitative analysis on the mixing width and circulations demonstrates the effects of initial conditions on the interface instability. The evolution principle of interface instability in the strongly nonlinear regime after reshock in the nonuniform flows is obtained. These results are expected to shed new light on the turbulent mixing induced by RM instability. 


\section{Governing equation, algorithm and MVFT code}

The numerical simulations have been done by our large eddy simulation code MVFT (multi-viscous flow and turbulent). The code MVFT can be used to simulate multi-component flows, and compute shocks, contact discontinuities and material interfaces at high accuracy.

MVFT applies the piecewise parabolic method [13] to interpolate physical quantities, the Vreman [14] subgrid eddy viscosity model to conduct the large eddy simulation, and solves the Navier-Stokes equations,

$$
\left\{\begin{array}{l}
\frac{\partial \bar{\rho}}{\partial t}+\frac{\partial \bar{\rho} \tilde{u}_{j}}{\partial x_{j}}=0 \\
\frac{\partial \bar{\rho} \tilde{u}_{i}}{\partial t}+\frac{\partial \bar{\rho} \tilde{u}_{j} \tilde{u}_{i}}{\partial x_{j}}+\frac{\partial \bar{p}}{\partial x_{i}}=\frac{\partial\left(\bar{\sigma}_{i j}+\tau_{i j}\right)}{\partial x_{j}} \\
\frac{\partial \bar{\rho} \bar{E}}{\partial t}+\frac{\partial\left(\bar{\rho} \tilde{u}_{j} \bar{E}+\bar{p} \tilde{u}_{j}\right)}{\partial x_{j}}=-\frac{\partial\left(\bar{q}_{j}+Q_{j}\right)}{\partial x_{j}}+\frac{\partial\left(\tilde{u}_{i}\left(\bar{\sigma}_{i j}+\tau_{i j}\right)\right)}{\partial x_{j}} \\
\frac{\partial \bar{Y}^{(s)}}{\partial t}+\tilde{u}_{j} \frac{\partial \bar{Y}^{(s)}}{\partial x_{j}}=\frac{\partial}{\partial x_{j}}\left(\tilde{D} \frac{\partial \bar{Y}^{(s)}}{\partial x_{j}}\right) s=1,2, \cdots, N-1
\end{array}\right.
$$

$\bar{\sigma}_{i j}=\mu_{l}\left(\partial \tilde{u}_{i} / \partial x_{j}+\partial \tilde{u}_{j} / \partial x_{i}-2 / 3 \delta_{i j}\left(\partial \tilde{u}_{k} / \partial x_{k}\right)\right)$ is the viscous stress tensor, $\tau_{i j}=\rho\left(\overline{u_{i} u_{j}}-\bar{u}_{i} \bar{u}_{j}\right)$ is the subgrid scale (SGS) stress tensor, $\bar{q}_{j}+Q_{j}$ is the energy flux of unit time and space, $\bar{q}_{j}=-\lambda_{l} \partial \bar{T} / \partial x_{j}, \quad Q_{j}=-\lambda_{t} \partial \bar{T} / \partial x_{j}$, $\lambda_{l}=\mu_{l} c_{p} / p_{r, l}, \quad \lambda_{t}=\mu_{t} c_{p} / p_{r, t}, \quad \tilde{D}=\bar{D}_{l}+D_{t}, \quad S_{c, t}=\mu_{t} / D_{t} \bar{\rho} . \quad \mu_{l}$ is the fluid viscosity, $\mu_{t}$ is the turbulent viscosity, $\bar{T}$ is the temperature, $\lambda_{l}$ is the efficient heat-transfer coefficient, $c_{p}$ is the specific heat of fluid, $p_{r, l}$ is the Prandtl number, $\bar{D}_{l}$ is the diffusion coefficient and $D_{t}$ is the turbulent diffusion coefficient. An operator splitting technique is used to decompose the physical problems into three sub-processes in MVFT, i.e., the computations of inviscid flux, viscous flux and heat flux. For the inviscid flux, the three-dimensional problem can be simplified into three $1 \mathrm{D}$ problems by dimension-splitting technique. For each 1D problem, we apply two-step Lagrange-Remap algorithm to solve the equations. The latter is calculated by utilizing a central difference scheme, in conjunction with a second order Runge-Kutta method. The numerical algorithms and SGS turbulent model have been presented in the author's literature [15].

\section{Numerical simulation and analysis}

The experimental gases are air and $\mathrm{SF}_{6}$, and the $\mathrm{SF}_{6}$ gas constitutes the initial nonuniform flow field. Figure 1 shows the initial structure diagram of shock tube experiment with $20 \times 10 \mathrm{~cm}^{2}$ rectangular cross section and computed domain. The incident shock wave mach number is 1.27 in air. The air shock wave through the 
air and $\mathrm{SF}_{6}$ interface, and enter into the $\mathrm{SF}_{6}$ gas nonuniform flow field. Two different kinds of initial sinusoidal perturbations with the same wavelength and different amplitude on the interface are set. The wavelength is $0.05 \mathrm{~m}$, the amplitudes are $5.0 \times 10^{-3} \mathrm{~m}$ and $7.5 \times 10^{-3} \mathrm{~m}$ corresponding to $0.0 \leq y<0.0875 \mathrm{~m}$ and $0.0875<y \leq 0.2 \mathrm{~m}$. The initial shock front is located at $x=5.56 \times 10^{-3} \mathrm{~m}$, the equilibrium position of perturbation is at $x=0.016 \mathrm{~m}$, the range of observation test window is $[0.038 \mathrm{~m}, 0.25 \mathrm{~m}]$ in the $x$ direction. Table I summarizes the properties of air and $\mathrm{SF}_{6}$ gas in the present experiment at 1 atmospheric pressure and $20^{\circ} \mathrm{C}$.

Table 1: $\quad$ Properties of air and $\mathrm{SF}_{6}$ gases.

\begin{tabular}{cccccc}
\hline \hline Gases & $\begin{array}{c}\text { Density } \\
\left(\mathrm{kg} / \mathrm{m}^{3}\right)\end{array}$ & $\begin{array}{c}\text { Specific } \\
\text { heat ratio }\end{array}$ & $\begin{array}{c}\text { Kinematic } \\
\text { viscosity } \\
\left(10^{-6} \mathrm{~m}^{2} / \mathrm{s}\right)\end{array}$ & $\begin{array}{c}\text { Prandtl } \\
\text { number }\end{array}$ & $\begin{array}{c}\text { Diffusion } \\
\text { coefficient } \\
\text { in air }\left(\mathrm{cm}^{2} / \mathrm{s}\right)\end{array}$ \\
\hline Air & 1.29 & 1.40 & 15.7 & 0.71 & 0.204 \\
$\mathrm{SF}_{6}$ & 5.34 & 1.009 & 2.47 & 0.90 & 0.097 \\
\hline \hline
\end{tabular}

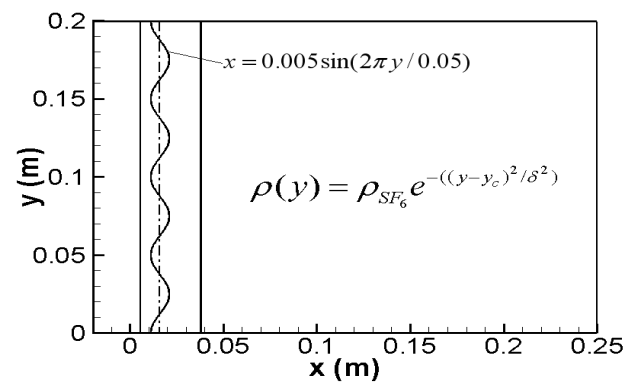

Figure 1: Initial structure diagram in the shock tube.

For the initial non uniform $\mathrm{SF}_{6}$ gas flow field, numerical simulation is used to approximately describe the dissipative transition layer [13]. In the dissipative transition layer, $\mathrm{SF}_{6}$ gas density is calculated by Gaussian function:

$$
\rho(y)=\rho_{S F_{6}} e^{-\left(\left(y-y_{c}\right)^{2} / \delta^{2}\right)}
$$

where $y_{\mathrm{c}}=0$, and $\delta=0.3729 \mathrm{~m}$. The calculating region is discretized into $540 \times 400$ grids. Sample images from the experiment and the corresponding numerical results are shown in Figure 2. It can be seen that, due to the nonuniform flow field of $\mathrm{SF}_{6}$ gas, the density distribution changes from high to low along the shock tube vertically, and this results in the propagating velocity of shock wave in the upper part of shock tube faster than in the bottom, and forms an oblique shock wave front. The calculated development of the interface shape, location, and oblique shock wave propagating features are consistent with the experimental results. Figure 3 shows that the calculated results of uniform flow are quite different from the nonuniform flow results. Figure 4 shows the 
comparison of location of three test lines at different times between experiment and simulation. The largest difference is about $5 \%$, this difference may be due to the initial approximation of the real situation. Figure 5 shows the comparison between perturbation amplitude of experiment, numerical computing, theoretical model, in which the bar denotes the experimental error about $\pm 10 \%$. We can see that, the difference between numerical and experimental values were within $10 \%$.

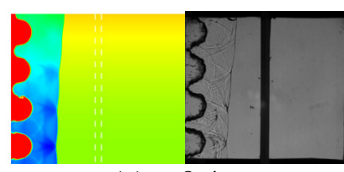

(a) $\mathrm{t}=0.4 \mathrm{~ms}$

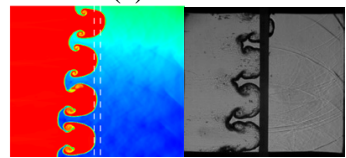

(c) $\mathrm{t}=1.2 \mathrm{~ms}$

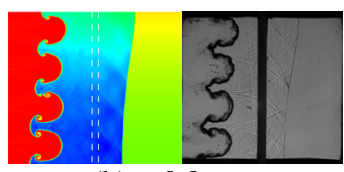

(b) $\mathrm{t}=0 . \overline{\mathrm{ms}}$

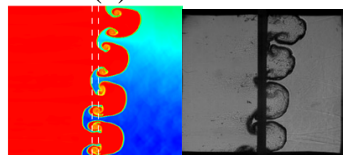

(d) $\mathrm{t}=1.6 \mathrm{~ms}$

Figure 2: Schlieren photography pictures (right column) and numerical simulation results by MVFT2D (left column) at a certain time.
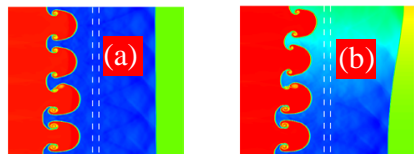

Figure 3: The difference of results at $\mathrm{t}=1.0 \mathrm{~ms}$ between the initial uniform and nonuniform flow ((a) Initial uniform flow (b) Initial non-uniform flow).

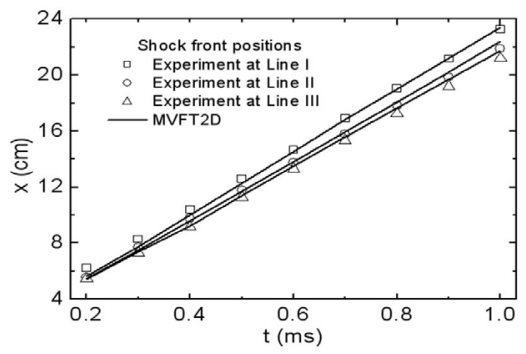

Figure 4: $\quad$ Shock front locations of the experiment and calculated results on the three test lines at different times. 


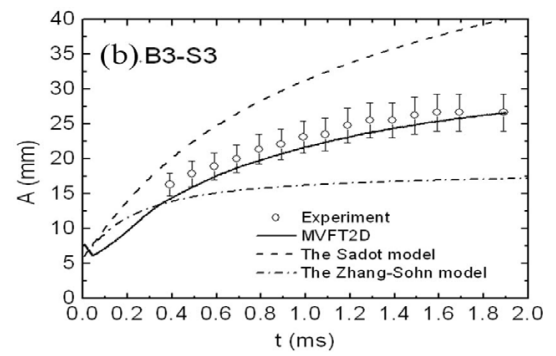

Figure 5: Perturbation amplitudes of the experiment, numerical computing, and comparison with the theoretical model.

In order to investigate the effect of the nonuniformity of flows on the evolution of instability, we perform the numerical study of the instability and mixing with the initial Gaussian distribution nonuniform flows of two different nonuniform coefficients $\left(\delta_{1}=0.6162\right.$, and $\left.\delta_{2}=0.4961\right)$ under reshocks. The initial Air/ $\mathrm{SF}_{6}$ interface for single-mode sinusoidal perturbation is that the wavelength is $\lambda=0.05 \mathrm{~m}$, and the amplitude is $5.0 \times 10^{-3} \mathrm{~m}$. The incident shock wave Mach number is 1.25 in air. The less the nonuniform coefficient is, the stronger the nonuniformity of the flow is.

Figure 6 shows the density contour images of the numerical results by MVFT at several times, the left, middle and right columns correspond with the uniform initial condition simulation results, the $\delta_{1}$ and $\delta_{2}$ nonuniform Gaussian function cases. Figure 6 indicates qualitatively that, the disturbed interface can preserve good periodicity before and after reshock. The nonuniformity resulted from the density variances of the $\mathrm{SF}_{6}$ gas in the nonuniform flows makes the transmitted and reflected shocks inclined. However, the simulation results show that there is a significant difference between the uniform and nonuniform flows before reshock, and the difference decreases in evidence after reshock.

To estimate the mixing width from the numerical simulations, we calculate the transversal averaged volume fraction $\bar{Y}(x)$ in each abscissa $x$, and define the abscissa $x$ between of the $\bar{Y}(x) 0.01$ and 0.99 as the mixing zone width. Figure 7 shows the mixing width history of the initial uniform and nonuniform flow in $\mathrm{RM}$ instability. It points out that the growth of the mixing width for the initial nonuniform flows is greater than the uniform flow, and the less the nonuniform coefficient is, the faster the growth of mixing width is, but the difference among the three different flow configurations diminishes after reshock. These results indicate that the evolution of the instability has great dependence on the nonuniformity of the initial flow in the linear and weakly nonlinear regime prior to reshock, however, the effect of the nonuniformity is reduced significantly with the instability entering the strongly nonlinear regime after reshock. Although the growth of the perturbation enhances, the amplitude of the perturbation is close to a uniform flow comparing with the previous regime. 


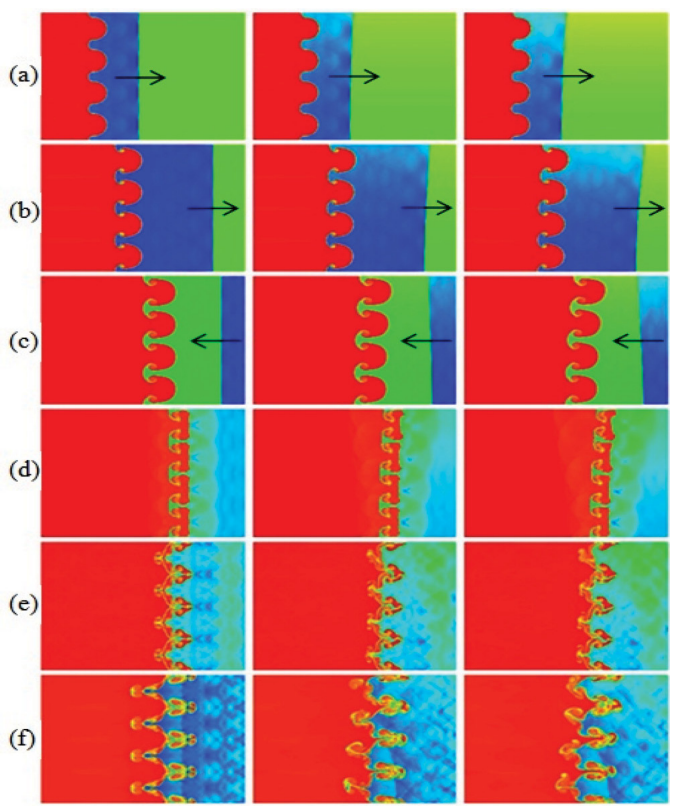

Figure 6: (Color online) Density contour images of the numerical simulation result by MVFT at a certain time (a) $0.5 \mathrm{~ms}$, (b) $1.0 \mathrm{~ms}$, (c) $1.5 \mathrm{~ms}$, (d) $2.0 \mathrm{~ms}$, (e) $2.5 \mathrm{~ms}$ and (f) $3.0 \mathrm{~ms}$. (The left column with uniform initial conditions, the middle column with a $\delta_{1}$ nonuniform Gaussian function and the right column with a $\delta_{2}$ nonuniform Gaussian function. The small arrow denotes the direction of propagation of the shock wave fronts before reshock the interface.).

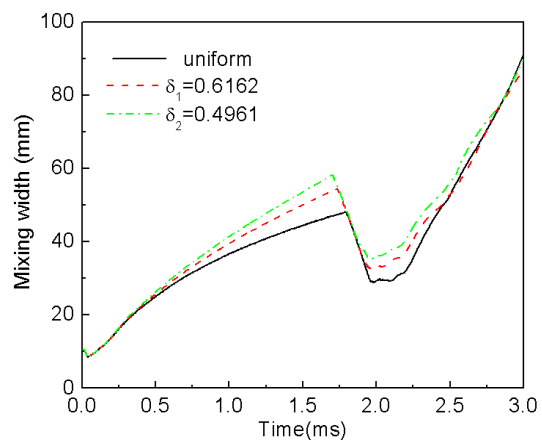

Figure 7: Mixing width history calculations of the initial uniform and nonuniform flow in RM instability. 
This evolution process of the mixing width is a macro description of numerical simulation to RM instability and mixing. To interpret the phenomena, the analysis of the underlying mechanism resulting in RM instability is required, namely the analysis of the effects of the vorticity deposited by the baroclinic torque production term and the circulation in the mixing zone induced by RM instability. The vorticity is determined by calculating the curl of the velocity field in 2D flow:

$$
\omega(x, y, t)=\nabla \times \mathbf{V}=\partial v / \partial x-\partial u / \partial y
$$

where $\mathbf{V}$ is the 2D velocity vector, and $u$ and $v$ are the $x$ and $y$ components of the velocity. Circulation is a measure of the average vorticity over an area $A$,

$$
\Gamma(t)=\int_{A} \omega(x, y, t) \cdot d A
$$

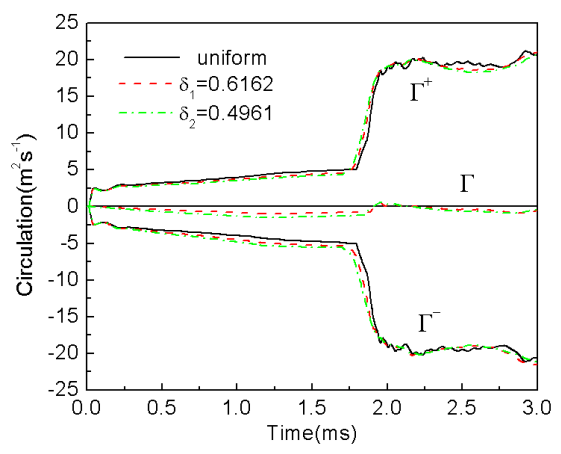

Figure 8: The positive circulation, the negative circulation and the total circulation evolution over time of the flow field of the two elliptic gas cylinders.

Figure 8 shows the positive circulation $\Gamma^{+}$, the negative circulation $\Gamma^{-}$and the total circulation $\Gamma=\Gamma^{+}+\Gamma^{-}$evolution over time of the flow field. In figure 8, the results indicate that $\Gamma^{+}$and $\Gamma^{-}$conserve the very good symmetry for the initial uniform flow, and the total circulation $\Gamma$ is 0 all the time. For the initial nonuniform flows, $\Gamma^{+}$and $\Gamma^{-}$no longer conserve symmetry due to the dissymmetry growth of the perturbation, and the total circulations of the flows $\Gamma$ are none zero. In order to further analyze the differences among three sets of curves in figure 8, the relative errors of the circulations (both positive $\Gamma^{+}$and negative $\Gamma^{-}$) between the two initial nonuniform and the uniform flows are calculated and presented in the figure 9(a) and (b), respectively. Figure 9(a) shows the maximum differences of $\Gamma^{+}$and $\Gamma^{-}$are $9.8 \%$ and $13.8 \%$ separately before reshock, the maximum differences reach $19.7 \%$ and $24.8 \%$ in the transition regime, and the maximum departures are only $5.6 \%$ and $4.8 \%$ after reshock for the flow with the nonuniform coefficient $\delta_{1}$. Figure 9(b) shows the maximum differences of $\Gamma^{+}$and $\Gamma^{-}$are $13.6 \%$ and $24.5 \%, 32.7 \%$ and $55.7 \%$, $7.3 \%$ and $4.0 \%$ before reshock, in the transition regime and after reshock 


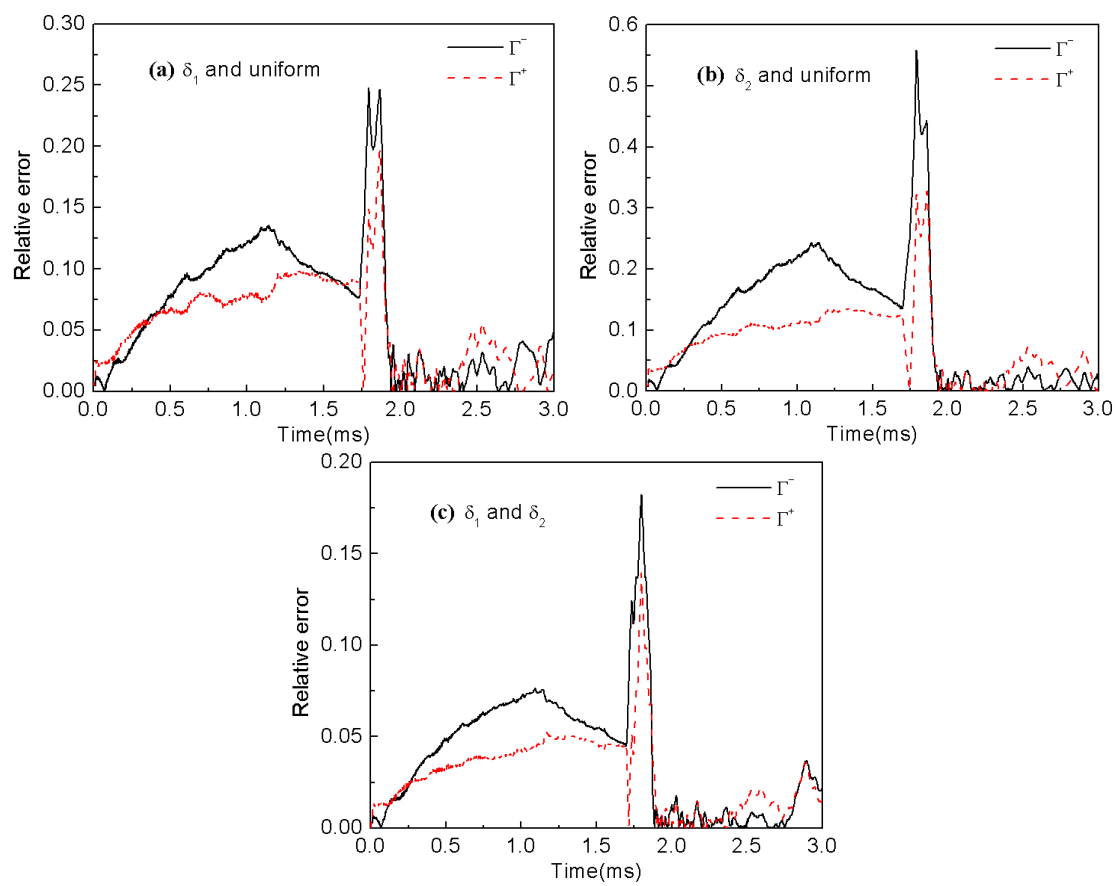

Figure 9: Relative error between the flow field in the positive and negative circulations, including the comparison between the nonuniform coefficients $\delta_{1}$ and $\delta_{2}$ with uniform flow (in Figure 7(a) and Figure 7(b) ) and the nonuniform coefficient between the $\delta_{1}$ and $\delta_{2}$ (in Figure $7(\mathrm{c})$ ). The reshock time is $1.73 \mathrm{~ms}$ and $1.70 \mathrm{~ms}$ and $1.70 \mathrm{~ms}$ respectively in the case of three graphical.

separately for the flow with the nonuniform coefficient $\delta_{2}$. These results indicate that the differences between the nonuniform coefficients comparing to the uniform flow exist prior to reshock and in the transition regime, and diminish significantly after reshock with the mean value 5\% approximately. Because the circulation in the flow exists in the interface zone, it affects the evolution of the mixing zone directly. In addition, the difference between two different initial nonuniform flows is also exhibited in figure 9(c) which shows that the maximum differences of $\Gamma^{+}$and $\Gamma^{-}$are $5.3 \%$ and $7.6 \%$ before reshock, $13.9 \%$ and $18.2 \%$ in the transition regime, $3.6 \%$ and $3.7 \%$ after reshock separately.

\section{Summary}

In summary, we numerically simulate $\mathrm{RM}$ instability and mixing of Air/SF 6 interface with sinusoidal perturbation under shocks and reshocks, by constructing two initial nonuniform density flows of Gaussian distribution 
function with different coefficients of $\delta_{1}=0.6162$ and $\delta_{2}=0.4961$. The distinctions of evolution and development of mixing zone between nonuniform cases and uniform one are analyzed. The results demonstrate that the evolution of the instability has a great dependence on the nonuniformity of the initial flow field in the linear and weak nonlinear regime prior to reshock. Nevertheless, the effect of the nonuniformity is reduced significantly as the instability enters the strongly nonlinear regime after reshock. Although is the growth of the perturbation is enhanced in this regime, the amplitude of the perturbation is close to that of a uniform flow comparing with the previous regime. The reason of the above mentioned phenomena is presented with the quantitative analysis of the circulation. In addition, the comparisons of the computational results between two initial nonuniform flows are shown. This paper further demonstrates that the effects of the initial conditions of flows on the macro scale characteristics are weakened gradually in the later time of RM instability. Naturally, we speculate that the flow field will completely forget the effects of the initial conditions in the fully developed regime, which can become turbulent.

\section{Acknowledgements}

The work was sponsored by the National Science Foundation of China Grant No. 11072228 and 11202195, the Science Foundation of China Academy of Engineering Physics Grant No. 2011B0202005 and 2011A0201002.

\section{References}

[1] J. D. Lindl, R. L. McCropy, E. M. Campbell, Phys. Today 45, 32 (1992).

[2] J. Yang, T. Kubota, E. E. Zukoski, AIAA J. 31, 5 (1993).

[3] D. Arnett, Astrophys. J. Suppl. Series 127, 213 (2000).

[4] R. D. Richtmyer, Commun. Pure Appl. Math. 13, 297 (1960).

[5] K. O. Mikaelian, Phys. Rev. E 67, 026319 (2003).

[6] Qiang Zhang, Sung-Ik Sohn, Z. Angew. Math. Phys. 50, 1 (1999).

[7] B. Thornber, D. Drikakis, D. L. Youngs, and R. J. R. Williams, Phys. Fluids 23, 095107 (2011).

[8] Oleg Schilling, Marco Latini, Wai Sun Don, Phys. Rev. E 76, 026319 (2007).

[9] D. J. Hill, C. Pantano, D. I. Pullin, J. Fluid Mech. 557, 29 (2006).

[10] S. Ukai, K. Balakrishnan, S. Menon, Shock Waves 21, 533 (2011).

[11] E. Leinov, O. Sadot, A. Formoza, G. Malamud, Y. Elbaz, L. A. Levin, G. Ben-Dor and D. Shvarts, Phys. Scr. T132, 014014 (2008).

[12] J. S. Bai, J. H. Liu, T. Wang, L. Y. Zou, P. Li, D. W. Tan, Phys. Rev. E 81, 056302 (2010).

[13] P. Colella and P. R. Woodward, J. Comput. Phys. 54, 174 (1984).

[14] W. Vreman, Phys. Fluids 16, 3670 (2004).

[15] J. S. Bai, L. Y. Zou, T. Wang, K. Liu, W. B. Huang, J. H. Liu, P. Li, D. W. Tan, C. L. Liu, Phys. Rev. E 82, 056318 (2010 ). 